Metbylnitrat der Kakothelinbase.

$1 \mathrm{~g}$ Methylbrucin wurde in $30 \mathrm{ccm}$ 10-proz. Salpetersäure gelöst und über freier Flamme bis zum Sieden erbitzt. Die Lösung färbte sich dabei plötzlich rot unter Entwicklung nitroser Gase und nach einige Minuten langem Kochen orange. Beim Abkühlen schieden sich orangefarbene Täfelchen aus, die nach dem Stehen in Eis abfiltriert, mit verdünnter Säure und Aceton gewaschen und an der Lnft getrocknet wurden. Ausbeute $0.7 \mathrm{~g}$. Die Einwirkung stark abgekühlter konzentrierter Salpetersäure liefert zwar das gleiche Produkt, aber in weit geringerer Menge.

$$
\begin{aligned}
& \mathrm{C}_{21} \mathrm{H}_{21} \mathrm{~N}_{3} \mathrm{O}_{7}, \mathrm{CH}_{3} . \mathrm{NO}_{3}+{ }^{3} \mathrm{H}_{2} \mathrm{O} . \\
& \text { Ber. C } 48.80, \quad \mathrm{H} 5.18, \quad \mathrm{~N} 10.35, \mathrm{H}_{2} \mathrm{O} 6.65 . \\
& \text { Gef. } \gg 49.16,48.57, 》 5.25,5.48, 》 10.46, \$ 6.60,6.37
\end{aligned}
$$

(bei $100^{\circ}$ i. V. u. $\mathrm{P}, \mathrm{O}_{5}$ ).

Im Capillarrohr erhitzt, wird das Salz allmählich dunkel und scheint gegen $280^{\circ}$, ohne zu schmelzen, völlig zu verkohlen. Rasch erwärmt, verpufft es noch leichter als Kakothelin. In salzsaurer Lōsung gibt es mit Schwefeldioxyd eine violette Lösung und nach einiger Zeit eine Abscheidung violetter Prismen.

400. Hermann Leuchs und Walter Gelger: Ober die Isolierung einer vierten Brucin-8ulfosture. XIII. Mittellung: uber Stryahnos-Alkaloide.

[Aus dem Chemischen Institut der Universitāt Berlin.]

(Eingegangen am 14. Oktober 1911.)

Die für die Bildung von Sulfosäuren des Brucins und Strychnins. mit’̉Braunstein und schwefliger Säure zuerst aufgestellte Theorie ') verlangte das $A$ uftreten von vier teils struktur-, teils stereoisomeren Formen. Der Eintritt der Sulfogruppe in das scbon asymmetrische und aktive Molekül der Alkaloide mußte zur Bildung von je zwei räumlich isomeren, aber nicht enantiomorphen Säuren führen, wenn

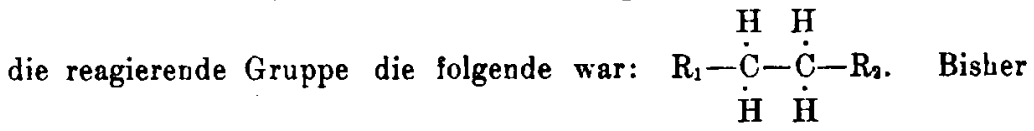
konnten jedoch beim Strychnin und Brucin nur drei Formen nachgewiesen werden, so daß die obige Gruppe durch Einfügung eines

1) B. 41, $4393[1908] ; 42,2681,3067$ [1909]. 
Restes $R_{3}$ diesem Umstand angepaßt wurde. Wir müssen nun aber doch die erste Formulierung als den zutreffenden Ausdruck ansehen, nachdem wir durch mühselige Versuche die Existenz einer vierten isomeren Brucin-sulfosäure sichergestellt haben.

\section{Brucin-sulfosüure IV.}

Die Reaktion zwischen Brucin, Braunstein und Schwefeldioxyd wurde genau so wie früher ausgefïhrt. Die Abscheidung und Trennung der Sulfosäuren verlief jedoch nicht stets in der gleichen Weise, da sie im wesentlichen auf einer fraktionierten Krystallisation berubt. So führte bisweilen das zur Reindarstellung der Säure I vorgeschriebene Umkrystallisieren aus heißem Wasser nicht zum Ziele, indem die Drehung nur auf etwa $-200^{\circ}$ statt $-242^{\circ}$ gebracht werden konnte. In diesen Fällen erwies sich dann Umlösen aus 13 Volumteilen 80-proz. Alkohol als sehr förderlich. Denn die hieraus sich abscheidenden, großen, massiven, quadratischen Tafeln von Säure I zeigten nach ein- bis zweimaliger Ausführung der Operation die richtige Drehung.

Bei einem derartigen Versuch blieben nun einige Prozent im heißen Sprit ungelöst. Diese Menge löste sich erst in 169 Teilen kochendem Wasser und fiel zum größten Teil in der Kälte in Form großer, glänzender, an den Enden abgeschrägter Prismen wieder aus. Ebenso wie durch die Löslichkeit und Krystallform unterschied sich diese sauer reagierende Substanz durch die optische Drehung von den bekannten drei Brucin-sulfosäuren:

$0.0593 \mathrm{~g}$ getr. Sbst. in $2.5 \mathrm{ccm}=/ 10^{-L a u g e ~(2 ~ M o l .) ; ~} 2.577 \mathrm{~g}$ Lösung: Proz.-Gehalt 2.30; spez. Gew. 1.01; Drehung im 0.5-dem-Rohr $-142^{\circ}$.

$$
[a]_{\mathrm{D}}^{20}=-122.2^{0} \text {. }
$$

Die lufttrockne Säure verlor bei $105^{0}$ im Vakuum über Phosphorpentoxyd Wasscr.

$\mathrm{C}_{23} \mathrm{H}_{26} \mathrm{O}_{7} \mathrm{~N}_{2} \mathrm{~S}+4 \mathrm{H}_{2} \mathrm{O}$. Ber. $\mathrm{H}_{2} \mathrm{O}$ 13.18. Gef. $\mathrm{H}_{2} \mathrm{O} 13.12$.

$0.1139 \mathrm{~g}$ getr. Sbst.: $0.2442 \mathrm{~g} \mathrm{CO}_{2}, 0.0571 \mathrm{~g} \mathrm{H}_{2} \mathrm{O} .-0.1676 \mathrm{~g}$ getr. Sbst.: $0.0808 \mathrm{~g} \mathrm{BaSO}_{4}$.

$$
\begin{aligned}
& \mathrm{C}_{28} \mathrm{H}_{26} \mathrm{O}_{7} \mathrm{~N}_{3} \mathrm{~S} \text {. Ber. C } 58.23, \mathrm{H} \text { 5.48, S } 6.75 \text {. } \\
& \text { Gef. \$ 58.47, } 5.62, \gg 6.62 \text {. }
\end{aligned}
$$

Auch die Säure II enthielt manchmal geringe Mengen der veuen Säure. Wir trennten das Gemisch durch Auskochen mit 150 Teilen 80-proz. Alkohol und identifizierten das Ungelöste (bis $5 \%$ ) durch seine Löslichkeit und Krystallform.

Einen Anbalt über die entstandene Menge gibt die folgende dritte Isolierung: 
Es wurden $120 \mathrm{~g}$ Brucin verarbeitet. Die Hauptmenge der Säuren (ca. $130 \mathrm{~g}$ ) schied sich nach kurzem Steben in Eis ab. Nach mehreren Tagen war eine $z$ weite, weit geringere Krystallisation erfolgt. Diese wurde zuerst mit $50 \mathrm{ccm}$ Wasser ausgekocht, die genügt bätten, vorhandene Säure I völlig zu lösen, dann noch mit $500 \mathrm{ccm}$ Wasser, die auch II und III hätten aufnehmen müssen. Es blieben jedoch $0.6 \mathrm{~g}$ der schwer löslichen Säure zurück.

Aus 200 Teilen Wasser umkrystallisiert und bei $78^{\circ}$ im Vakuum getrocknet (Gew.-Verl. 12.78\%), zeigte sie unter den oben angegebenen Bedingungen $[a]_{\mathrm{D}}^{20}=-119.8^{\circ}$. Nach dem Auskochen mit 10 Teilen Wasser und abermaligem Umlösen war $[\alpha]_{D}^{20}=-119.2^{\circ}$. Wiederholtes Umkrystallisieren gab eine Substanz mit einem Wassergehalt in lufttrocknem Zustande von $13.11 \%$ und mit $[\alpha]_{\mathrm{D}}^{20}=-122.5^{\circ}$.

Durch das Auskochen der zweiten Krystallisation mit $550 \mathrm{ccm}$ Wasser sind etwa $3 \mathrm{~g}$ Săure IV entfernt worden. Sie entsteht also mindestens in einer Menge von $3.6 \mathrm{~g}$ aus $120 \mathrm{~g}$ Brucin.

Die folgende Zusammenstellung gibt einen Vergleich der Löslichkeit in kochendem Wasser und der Drehung der vier Säuren:

$\begin{array}{lll}\text { Sãure I: } & 1: 12 & {[a]_{\mathrm{D}}^{20}=-242^{\circ},} \\ \text { Säure II: } & 1: 55 & {[\alpha]_{\mathrm{D}}^{20}=+29^{\circ},} \\ \text { Säure III: } & 1: 55 & {[\alpha]_{\mathrm{D}}^{20}=+157^{\circ},} \\ \text { Såure IV: } & 1: 170 & {[\alpha]_{\mathrm{D}}^{20}=-122^{\circ} .}\end{array}$

401. A. Windaus und J. Adamla: Ober Oholesterglamin. XIII. Mittellung: Zur Kenntnis des Oholesterins.

[Aus der Medizinischen Abteilung des Universitätslaboratoriums Freiburg i. B.] (Eingegangen am 17. Oktober 1911.)

Über das Cbolesterylamin, $\mathrm{C}_{27} \mathrm{H}_{45} . \mathrm{NH}_{2}$, das Amin der CholesterinReibe, hat $\mathrm{L}_{\text {oebis }} \mathrm{ch}^{1}$ ) im Jahre 1872 eine kurze Mitteilung veröffentlicht. Er behauptet, er habe das Cholesterylchlorid durch Erhitzen mit alkoholischem Ammoniak auf $100^{\circ}$ in Cholesterylamin übergeführt und beschreibt diese Base als eine in irisierenden Blättchen vom Schmp. $104^{\circ}$ krystallisierende Substanz, die beim Abküblen aus der Schmelze ähnlich wie Cholesterylchlorid ein leuchtend blauviolettes

?) B. 5, 514 [1872]. 